\title{
minarit
}

Revista Educación

ISSN: 0379-7082

ISSN: 2215-2644

revedu@gmail.com

Universidad de Costa Rica

Costa Rica

\section{Actitud del estudiantado universitario hacia la revisión colaborativa de errores}

Ortiz Navarrete, Mabel; Salazar Betancur, Lorena

Actitud del estudiantado universitario hacia la revisión colaborativa de errores ${ }^{1}$

Revista Educación, vol. 46, núm. 1, 2022

Universidad de Costa Rica, Costa Rica

Disponible en: https://www.redalyc.org/articulo.oa?id=44068165023

DOI: https://doi.org/10.15517/revedu.v46i1.45197

\section{(c) $(1) \Theta(\Theta$}

Esta obra está bajo una Licencia Creative Commons Atribución-NoComercial-SinDerivar 3.0 Internacional. 


\title{
Actitud del estudiantado universitario hacia la revisión colaborativa de errores ${ }^{1}$
}

\author{
Undergraduate Student Attitudes Regarding Collaborative Error Revisions ${ }^{2}$
}

Mabel Ortiz Navarrete

Universidad Católica de la Santísima Concepción, Chile

mortiz@ucsc.cl

iD https://orcid.org/0000-0001-7481-1293

\author{
Lorena Salazar Betancur \\ Universidad Católica de la Santísima Concepción, Chile \\ lsalazar@gmail.com \\ iD https://orcid.org/0000-0002-2856-5113
}

DOI: https://doi.org/10.15517/revedu.v46i1.45197
Redalyc: https://www.redalyc.org/articulo.oa?
id=44068165023

\section{Resumen:}

El presente artículo tiene como objetivo analizar la actitud de un grupo de futuros profesores y profesoras de inglés hacia la revisión colaborativa de errores gramaticales cuando escriben un ensayo argumentativo. El estudio sigue un diseño descriptivo no experimental cuya muestra estuvo constituida por 29 estudiantes de Pedagogía Media en Inglés de una universidad chilena. Para la recolección de datos se utilizaron dos instrumentos: una escala Likert y una entrevista semiestructurada. Los datos cualitativos fueron analizados a través de la técnica de análisis de contenido, que permitió identificar categorías y subcategorías. Los resultados evidencian que existe en general una valoración positiva hacia la revisión colaborativa de errores gramaticales. De acuerdo con las opiniones de los/las estudiantes, la revisión colaborativa de errores gramaticales contribuye a la discusión y a la disipación de dudas, a promover la autonomía y a facilitar el aprendizaje. Los resultados de este estudio pueden servir para investigaciones futuras que se lleven a cabo en el contexto de aprendizaje de una segunda lengua.

Palabras ClaVe: Actitud del estudiantado, Enseñanza de segunda lengua, Gramática, Revisión colaborativa de errores, Errores gramaticales.

\section{AbstraCt:}

This study analyzes pre-service English Language Education instructors regarding the identification of grammatical errors and collaborative revisions made to argumentative essays. The study encompasses a non-experimental descriptive design based on a sample group of 29 English Education students from a Chilean university. Data was collected using a Likert scale and semistructured interviews. Qualitative data was analyzed through content analysis techniques which allowed quantitative data to be classified according to categories and subcategories. Results show that there is a positive attitude towards collaborative revision of grammatical errors. According to the opinion of the participating students, collaborative revision of errors promotes autonomy, facilitates learning and contributes to discussion as well as contributes to resolving doubts. The results of this study can be useful for future research conducted within the context of foreign language instruction.

KEYWORDS: Student Attitudes, Foreign Language Instruction, Grammar, Collaborative Error Revisions, Grammatical Errors.

\section{INTRODUCCIÓN}

El aprendizaje de una segunda lengua conlleva un proceso complejo, puesto que implica el desarrollo de competencias lingüísticas y comunicativas, a las que, además, se suman otros factores, tales como: motivación y diferencias estructurales entre una lengua y otra, en el contexto de este artículo, las diferencias entre el idioma inglés y el idioma español en Chile. En este sentido, el aprendizaje del inglés en Chile es considerado problemático. El tema de la precisión lingüística o accuracy que, si bien puede no interferir en la comunicación de un mensaje, sí se convierte en una problemática que requiere atención en el futuro profesorado de inglés. 
De acuerdo a información del Ministerio de Educación (Mineduc, 2014), "los estándares disciplinarios de carreras de pedagogía en inglés postulan que los/las egresados/as deben comunicarse de forma precisa y fluida, en todos los ámbitos en los que les corresponde actuar, a nivel C1" (p.16). Por ello, centrarse en aspectos de forma escrita resulta esencial, ya que son considerados parte fundamental en el perfil del futuro profesorado de inglés.

Dentro de la formación pedagógica actual, los nuevos enfoques de enseñanza, tales como, el enfoque comunicativo, el enfoque basado en tareas, entre otros, han llevado a un cambio en el rol del profesorado y del estudiantado dentro del aula. Este último ha logrado desarrollar un papel cada vez más protagónico y autónomo. En este contexto, una metodología que, dada sus características, se presenta como una herramienta de gran utilidad en la corrección de errores gramaticales frecuentes y que resultan complejos de tratar individualmente, es la colaborativa. Esta metodología puede facilitar la corrección de errores dado que permite la disipación de dudas a través de la discusión, donde el grupo de estudiantes con mayores habilidades y conocimientos puede apoyar a la población estudiantil más débil.

A través de esta metodología, el estudiantado intenta resolver problemas de forma conjunta, a diferencia de una metodología en la que la persona docente asume un rol expositivo y el alumnado un rol netamente receptivo. Al respecto, algunas personas autoras consideran que, a través del aporte individual de cada estudiante, el grupo es capaz de escalar hacia un conocimiento nuevo que va más allá del que puede lograr individualmente (Baker, 2015; Li y Storch, 2017; Li y Zhu, 2017; Zhang, 2018).

El presente estudio se plantea como objetivo identificar la actitud de un grupo de futuro personal docente de inglés hacia la revisión colaborativa de errores gramaticales cuando escriben un ensayo argumentativo en inglés.

\section{REVISIÓN DE LA LITERATURA}

\section{Los errores en una segunda lengua}

El concepto de error ha sido ampliamente tratado en la literatura especializada enfocada en la adquisición de segundas lenguas. Blanco (2002) describe un error como "una transgresión, desviación o uso incorrecto de una norma, que en el caso que nos ocupa puede ser lingüística, pero también cultural, pragmática, y de una gran variedad de tipos más" (p. 20). Balcarcel (2006), por su parte, caracteriza un error "como un fenómeno sistemático y significativo en el proceso de aprendizaje" (p. 2). A su vez, Ellis (2009) lo define como una desviación de las normas o formas correctas de una L2. Ferris (2013) plantea que "una forma común de priorizar un error es focalizarse en aquellos errores que los/las estudiantes cometen frecuentemente" (p. 88). Por lo tanto, para efectos del presente estudio, se entenderá por error a aquel que ocurre con mayor frecuencia en un texto escrito.

Los errores gramaticales que comete el estudiantado son de diversa índole y muchos de ellos se relacionan con la interferencia de la lengua materna (Ocando y Urdaneta, 2015). En el contexto chileno, algunos de los errores más comunes son la $-s$ de la tercera persona, el uso de auxiliares en preguntas y negaciones, el uso de preposiciones, entre otros.

Una forma común de tratar estos errores es a través de distintos tipos de estrategias de feedback correctivo, que es generalmente entregado por el profesorado (Nassaji, 2017). Sin embargo, la provisión de feedback a veces no es suficiente, puesto que el estudiantado continúa cometiendo los mismos tipos de errores. Por esto se hace necesario buscar nuevas metodologías de corrección de errores que puedan apoyar el feedback que entrega el profesorado; una de ellas es la metodología colaborativa. 


\section{La metodología colaborativa}

La metodología colaborativa tiene como objetivo maximizar, a través de distintas técnicas y estrategias, los beneficios de la cooperación entre estudiantes (Assbeihat, 2016; Barkley et al., 2014; Baker, 2015; Pardo y Carrillo, 2016). Dichas técnicas y estrategias permiten que el trabajo colaborativo se desarrolle de manera más efectiva. En este aspecto, Barkley et al. (2014) señalan que existen tres requisitos para que el trabajo colaborativo sea efectivo. Primero, este debe tener un diseño intencional, organizado por el profesorado a través de tareas pre-estructuradas. Segundo, cada estudiante debe comprometerse a trabajar conjuntamente para alcanzar sus objetivos. Y tercero, el trabajo debe desarrollarse sobre una enseñanza significativa. Una vez cumplidos estos requisitos, el trabajo colaborativo provocará que el estudiantado ejercite su pensamiento, de tal manera que no solo se limiten a recibir información de las personas educadoras de manera pasiva.

Dentro de la metodología colaborativa, como muestra la Figura 1, existen tres elementos clave a considerar:

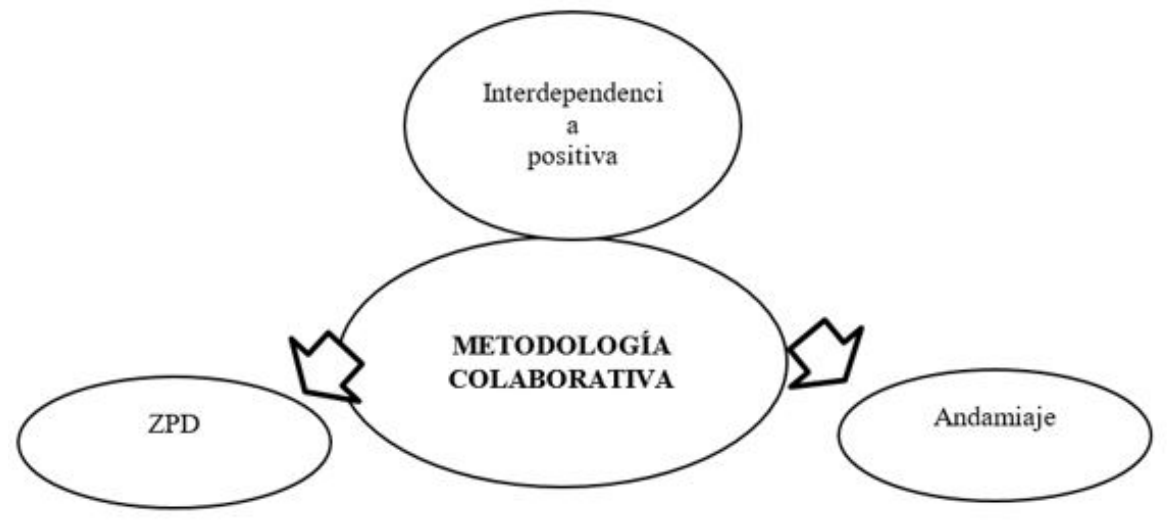

FIGURA 1.

Elementos de la metodología colaborativa

Fuente: elaboración propia.

La ZDP (Zona del Desarrollo Próximo) es definida como la distancia entre el nivel actual de desarrollo del alumnado y su nivel de desarrollo potencial (Vygotsky, 1978). La persona estudiante que presenta menos habilidades en L2 recibe asistencia de otra con habilidades superiores. Según Vygotsky (1978), esta instancia brindaría un mayor nivel de desarrollo cognitivo para el estudiantado, siempre que la interacción ocurra entre una persona experta y una novata de la L2. El segundo elemento es el andamiaje, concepto acuñado por Bruner $(1980,1983)$ citado en Delmastro (2008) para referirse "al apoyo de carácter intencional y transitorio que los compañeros y los adultos pueden proporcionar en situaciones específicas de enseñanzaaprendizaje" (p. 199); este consiste en la asistencia brindada por personas expertas a novatas a través de la interacción social, y que considera que el desarrollo del humano está situado naturalmente dentro de una actividad social. El andamiaje se da naturalmente cuando integrantes del grupo que trabaja colaborativamente posee destrezas variadas. Finalmente, otro elemento importante es la interdependencia positiva, entendida como "la relación que se establece entre los integrantes de un grupo para compartir procesos y resultados, lo que no significa que en todo momento de la clase permanezcan trabajando en equipo" (Ferreiro, 2004, p. 163). Quienes integran un grupo dependen mutuamente para el logro de las metas. Para que esta interdependencia sea positiva, se sugiere que el estudiantado adquiera distintos roles con distintas responsabilidades. 


\section{La corrección colaborativa de errores}

Hyland (2000) señala que durante la corrección colaborativa de errores el estudiantado participa de forma más activa, lo que a su vez le da más autonomía en su proceso de aprendizaje. La tarea de revisión colaborativa posee diversas ventajas. La más relevante es que es una actividad que ayuda a la identificación de los errores lingüísticos, esto mediante el diálogo que se genera entre estudiantes para poder corregir de manera conjunta (Swain, 2000). Lo anterior es posible a través de la discusión y negociación de ideas. A su vez, es importante destacar que el trabajo colaborativo permite que el estudiantado sea protagonista de su propio aprendizaje y, en este específico contexto, del proceso de revisión, tarea que tradicionalmente es llevada a cabo por el profesorado.

En cuanto a la actitud de la población estudiantil hacia este tipo de tareas, en un estudio llevado a cabo por Fernández y Blum (2013), el estudiantado señaló que este tipo de trabajo permitió poner más foco en la gramática, además, de practicar la segunda lengua de forma oral con sus compañeros y compañeras. Del mismo modo, Storch (2017) declara que para las personas que entrevistó la corrección de errores resultó ser extremadamente útil en el aprendizaje de vocabulario y en el desarrollo de la gramática.

\section{Metodología}

\section{Pregunta de investigación}

¿Cuáles son las actitudes del estudiantado hacia la revisión colaborativa de errores gramaticales?

Diseño

Se aplicó un diseño descriptivo no experimental, basado en la observación y posterior análisis del desarrollo de diversas variables en su hábitat natural (Hernández et al., 2014).

\section{Muestra}

Se utilizó una muestra no probabilística intencionada de treinta sujetos. En este tipo de muestra, la persona investigadora selecciona intencionalmente a quienes participarán en la investigación (Hernández et al., 2014).

\section{Participantes}

El grupo de participantes se compuso de 29 estudiantes de la asignatura de Lingüística Aplicada, pertenecientes a la carrera de Pedagogía Media en inglés de una universidad de Concepción, Chile. Las edades fluctuaban entre los 19 y 22 años y el estudiantado contaba con un nivel de inglés B1, de acuerdo al Cambridge English Test.

\section{Instrumentos}

Entrevista semi-estructurada: se diseñó una entrevista semiestructurada que contenía siete preguntas agrupadas en una sola dimensión y que apuntan a determinar la actitud del estudiantado hacia la corrección colaborativa de errores. Cuestionario: se utilizó una escala Likert con cinco niveles (Muy en desacuerdo, Desacuerdo, Neutro, De acuerdo, Muy de acuerdo) y nueve afirmaciones, a fin de conocer la actitud que el alumnado tiene hacia la corrección colaborativa de errores (Hernández et al., 2014). El objetivo de este instrumento era complementar la información entregada a través de la entrevista semiestructurada.

\section{Análisis de la información}

La información cualitativa fue analizada a través de la técnica de análisis de contenido, que es definida como una metodología que es utilizada para identificar unidades de análisis, codificar los datos y levantar categorías y subcategorías (Hernández et al., 2014). La Tabla 1 presenta las etapas del análisis de contenido llevado a cabo. 
TABLA 1

Etapas análisis de contenido

\begin{tabular}{|c|c|}
\hline Fases & Descripción \\
\hline Etapa 1 & Cada entrevista fue grabada y transcrita \\
\hline Etapa 2 & $\begin{array}{l}\text { Se importaron las entrevistas al Programa Vivo 10. S Se crearon nodos a } \\
\text { partir de categorías previamente establecidas que emergieron del marco teórico. } \\
\text { Se arrastraron unidades del texto, que poseian cualidades similares a cada } \\
\text { uno de los nodos creados. } \quad \text { Una vez alojadas las unidades del texto en el nodo } \\
\text { respectivo, se procedió a realizar una lectura de estas mismas para cerciorarse de } \\
\text { que correspondieran al nodo seleccionado. . Se procedió a precisar los nodos } \\
\text { por conceptos más específicos. }\end{array}$ \\
\hline
\end{tabular}

\begin{tabular}{|c|c|}
\hline Etapa 3 & $\begin{array}{l}\text { En esta fase se realizó una revisión de los datos con sus respectivas } \\
\text { categorías. Se solicitó a dos personas académicas que realizaran su propio } \\
\text { análisis. Para este fin, se compartió a cada persona evaluadora el documento con } \\
\text { cada categoria identificada y sus respectivas unidades de análisis. Este grupo } \\
\text { debia corroborar que las categorias identificadas representaran los fragmentos } \\
\text { (frases, ideas) tomados de las entrevistas. }\end{array}$ \\
\hline
\end{tabular}

Fuente: elaboración propia.

\section{Procedimiento de trabajo}

El estudio se llevó a cabo a través de las siguientes etapas:

\section{Etapa 1}

El estudio se inicia con la selección de la muestra. Dicha muestra fue intencionada, dado que las personas participantes fueron elegidas de acuerdo a criterios de la persona investigadora. En este contexto, la muestra estuvo constituida por veintinueve estudiantes de segundo año de una carrera universitaria destinada a la formación de profesores y profesoras de inglés en Chile. En esta etapa se procedió también al diseño de los instrumentos que fueron utilizados posteriormente para la recolección de datos.

\section{Etapa 2}

Se llevó a cabo una experiencia en la asignatura de Lingüística Aplicada, la cual consistió en la escritura colaborativa de un ensayo argumentativo. Durante este proceso, se asignaron roles de edición y se promovió la revisión colaborativa de errores gramaticales, con el fin de que el estudiantado discutiera y disipara dudas en conjunto.

\section{Etapa 3}

En la etapa final del estudio, con el fin de dar respuesta a la pregunta de investigación, se procedió a la recolección de la información a través de la aplicación de un cuestionario y de una entrevista semiestructurada.

\section{Resultados}

\section{Resultados entrevista semiestructurada}

La Tabla 2 ilustra la categoría Ventajas de la corrección colaborativa de errores y sus respectivas categorías y evidencias. 
TABLA 2

Categoría: Desventajas de la corrección colaborativa de errores

\begin{tabular}{|c|c|}
\hline Subcategoría & Evidencia \\
\hline Facilita la corrección & $\begin{array}{l}\text { "[... si alguien se equivoca puede que alguien que tenga } \\
\text { más experiencia en eso puede desarrollar esa parte, cada } \\
\text { uno puede aplicar su propia experiencia para los puntos } \\
\text { fuertes de cada uno, o sea, como harta inteligencia } \\
\text { mezclada" (sujeto 11, } 20 \text { julio de 2020). }\end{array}$ \\
\hline Promueve la interdependencia positiva & $\begin{array}{l}\text { "[...] siempre va a haber uno que es mejor que otro y otro } \\
\text { que es peor que otro, entonces cuando hay algo } \\
\text { equivocado, entre todos, ese mejor le va a enseñar a los } \\
\text { otros por qué está malo y los que estén en peor nivel van } \\
\text { a aprender más" (sujeto } 8,20 \text { de julio de } 2020 \text { ). }\end{array}$ \\
\hline Contribuye a disipar dudas & $\begin{array}{l}\text { "[...] yo creo que he mejorado bastante con los trabajos } \\
\text { que hemos tenido porque podemos trabajar } \\
\text { mutuamente, podemos corregirnos unos con otros y } \\
\text { disipar dudas" (sujeto } 12,20 \text { de julio de } 2020 \text { ). }\end{array}$ \\
\hline Facilita la discusión & $\begin{array}{l}\text { "[...] es diferente preguntarle a un profe, asi como profe, } \\
\text { ¿qué se hace aqui? o ccómo es esto?, porque la relación es } \\
\text { más distante, en cambio, con amigos, es más fácil la } \\
\text { discusión" (sujeto } 1,20 \text { de julio de } 2020 \text { ). }\end{array}$ \\
\hline Facilita el aprendizaje & $\begin{array}{l}\text { "[...] podemos construir el aprendizaje y el conocimiento } \\
\text { de una forma colaborativa, ya que muchas veces } \\
\text { cuando uno trata de aprender solo, comete errores y no } \\
\text { tiene a quién preguntarle" (Sujeto } 12,20 \text { de julio de } \\
2020 \text { ). }\end{array}$ \\
\hline Optimiza el tiempo de trabajo & $\begin{array}{l}\text { "[...] Si tengo a otra persona que tenga los conocimientos } \\
\text { en los cuales no soy bueno, yo creo que eso hace que los } \\
\text { trabajos sean más rápidos, sean mejores" (sujeto } 11,20 \\
\text { de julio de } 2020 \text { ). }\end{array}$ \\
\hline Promueve la autonomía del estudiantado & $\begin{array}{l}\text { "[...] el profesor da pistas para encontrar el error, para } \\
\text { que el alumno se dé cuenta por sí solo lo que de verdad } \\
\text { está malo, porque eso es más significativo" (Sujeto } 8,20 \\
\text { de julio de } 2020 \text { ) "El profesor nos deja ser más. Tiene } \\
\text { un rol más de observador y nosotros somos más } \\
\text { independientes." (sujeto } 4,20 \text { de julio de } 2020 \text { ). }\end{array}$ \\
\hline
\end{tabular}

Fuente: elaboración propia.

La Tabla 3 ilustra la categoría Desventajas de la corrección colaborativa de errores y sus respectivas categorías y evidencias. 
TABLA 3

Categoría: Desventajas de la corrección colaborativa de errores

\begin{tabular}{ll}
\hline Subcategoría & Evidencia \\
\hline Nivel de competencia gramatical & $\begin{array}{l}\text { "[...] todo depende de la persona con quien trabajas, porque si la persona tiene } \\
\text { menos vocabulario que tú, obviamente no va a ser como un trabajo muy positivo } \\
\text { o se va a demorar más" (Sujeto 7, 20 de julio de 2020). }\end{array}$ \\
\hline Grado de confianza entre pares & $\begin{array}{l}\text { "[...] requiere mucha confianza de los demás y aquellos que no son capaces de } \\
\text { aportar mucho, pasan a ser como un punto en contra, pasan a dificultar a ambos } \\
\text { el trabajo" (sujeto 11, 20 de julio de 2020). }\end{array}$
\end{tabular}

Fuente: elaboración propia.

\section{Resultados Escala Likert}

La Tabla 4 presenta las respuestas del estudiantado a cada afirmación.

TABLA 4.

La corrección colaborativa de errores gramaticales

\begin{tabular}{|c|c|c|c|c|c|c|}
\hline & Item & Totalmente en desacuerdo & Desacuerdo & Neutral & De acuerdo & Totalmente de acuerdo \\
\hline 1 & Es útil & 0 & 0 & 0 & 9 & 20 \\
\hline 2 & Es útil cuando el estudiantado posee distintos niveles de competencia gramatical en inglés & 0 & 1 & 3 & 7 & 18 \\
\hline 3 & Les permite aclarar dudas & 0 & 0 & 3 & 5 & 21 \\
\hline 4 & Les permite aprender de sus pares & 0 & 0 & 0 & 6 & 23 \\
\hline 5 & Puede ser más efectiva que la corrección individual & 0 & 0 & 4 & 9 & 16 \\
\hline 6 & Puede ser tan efectiva como la corrección del profesorado & 0 & 7 & 8 & 9 & 5 \\
\hline 7 & Ayuda a fortalecer la competencia gramatical en inglés & 0 & 1 & 1 & 15 & 12 \\
\hline 8 & Ayuda a reducir los errores & 0 & 1 & 8 & 8 & 12 \\
\hline 9 & Debe ser una actividad a fomentar en las clases de inglés & 0 & 0 & 1 & 11 & 17 \\
\hline
\end{tabular}

Fuente: elaboración propia.

En la Tabla 4 se puede observar que las respuestas del estudiantado se distribuyen principalmente entre la valoración neutral y totalmente de acuerdo. En relación al ítem es útil, de una muestra de 29 estudiantes, 20 declaran estar totalmente de acuerdo (69\%) y 9 de acuerdo (31\%). A su vez, en el ítem es útil cuando el estudiantado posee distintos niveles de competencia gramatical en inglés, se observa que 18 estudiantes declaran estar totalmente de acuerdo (62\%), 7 de acuerdo (24\%), 3 neutral (10\%) y 1 en desacuerdo (3\%).

Con respecto al ítem les permite aclarar dudas, se aprecia que 21 estudiantes declaran estar totalmente de acuerdo (72\%), 5 de acuerdo (17\%) y 3 neutral (10\%). En el ítem les permite aprender de sus pares, 23 estudiantes declaran estar completamente de acuerdo (69\%) y 6 de acuerdo (31\%). En relación al ítem puede ser más efectiva que la corrección individual, 16 estudiantes están totalmente de acuerdo (55\%), 9 de acuerdo (31\%) y 3 neutral (14\%).

Respecto al ítem puede ser tan efectiva como la corrección del profesor, 5 declaran estar totalmente de acuerdo (17\%), 9 de acuerdo (31\%), 8 neutral (28\%) y 7 en desacuerdo (24\%). En el ítem ayuda a fortalecer la competencia gramatical en inglés, 12 se muestran totalmente de acuerdo (41\%), 15 de acuerdo (52\%), 1 neutral (3\%) y 1 en desacuerdo (3\%). A su vez, en el ítem ayuda a reducir los errores, se observa que 12 estudiantes declaran estar totalmente de acuerdo (41\%), 8 de acuerdo (28\%), 8 neutro (28\%) y 1 en desacuerdo (3\%). Finalmente, en el ítem debe ser una actividad a fomentar en las clases de inglés, 17 estudiantes afirman estar totalmente de acuerdo (59\%), 11 de acuerdo (38 \%) y 1 neutral (3\%). 


\section{Discusión}

Como punto de partida, en relación con la opinión brindada por el grupo de estudiantes en la entrevista semiestructurada, se puede señalar que de manera general las personas entrevistadas declaran tener una actitud positiva hacia el trabajo colaborativo. De acuerdo con las opiniones brindadas, esta metodología cumple un rol importante en la disipación de dudas. Comúnmente sucede que, en el trabajo individual, no siempre se logra detectar errores y estos pasan desapercibidos; en cambio, en grupo siempre existirá una persona que detecte un error que las demás no notaron. Lo anterior se evidencia en la siguiente respuesta:

En el trabajo individual uno no ve todos los errores, independiente de que sea un trabajo tuyo o de otra persona y el hecho del otro darse cuenta; por ejemplo, al principio de una oración que esta mala, entonces tu te das cuenta que [sí] puede ser y lo analizas y te das cuenta que había más errores todavía (sujeto 10, 20 de julio de 2020).

$\mathrm{Al}$ respecto, Swain (2000) manifiesta que las tareas de tipo colaborativa, las cuales son por naturaleza de índole comunicativa y que estimulan la producción de la L2, fomentan la capacidad del estudiantado de notar los errores del lenguaje, lo que permite un aprendizaje efectivo de la L2. Es decir, luego de variadas actividades de trabajo colaborativo, se logra que el estudiantado desarrolle la capacidad de notar los errores que pueden cometer al escribir en la L2, lo que conlleva un aprendizaje de esta L2 a través del uso de esta misma. De igual forma, Ellis (2009) propone que la corrección de errores a través del feedback permite que el alumnado sea capaz de notar la brecha que existe entre la producción incorrecta que puedan realizar y la versión correcta de la lengua meta. Esto resulta ser de gran importancia para las personas estudiantes en el aprendizaje de una L2, puesto que, a través de la colaboración y la interdependencia positiva que se logra, pueden adquirir un mejor manejo de la L2. Esto se ve reflejado en la siguiente evidencia:

"[...] nos cooperamos y muchas veces yo sé algunas cosas que mi compañero no y viceversa, esa colaboración mutua, ese complemento es importante' (sujeto 2, 20 de julio de 2020).

Otro de los aspectos que el estudiantado considera significativo es la autonomía que el trabajo colaborativo logra brindarles. El grupo de estudiantes ya no cumple un rol de receptor en el aula, sino que adquiere un rol activo. Esto queda reflejado en la siguiente evidencia:

"[...] los profesores nos dan tiempo de que nos corrijamos entre nosotros y eso igual nos ayuda para poder identificar los errores por nosotros mismos, poder revisarle a alguien sus errores y ver que errores uno puede identificar" (sujeto 6, 20 de julio de 2020).

Lo anterior es corroborado por Miao et al. (2006), quienes declaran que a través del trabajo colaborativo de dar y recibir feedback de y para sus compañeros, las personas estudiantes logran desarrollar su autonomía.

De igual forma, las personas entrevistadas destacan el apoyo que pueden brindar sus pares, dado que este tipo de trabajo no solo les permite conocer las opiniones de sus compañeras y compañeros, sino que también les permite aprender a trabajar en equipo, a ser más tolerantes y a confiar en sus pares. Todos estos elementos en conjunto ayudan a lograr mejores resultados. Esto se refleja en la siguiente evidencia:

"[...] nos permite trabajar colaborativa, no solo desarrollar habilidades individuales, sino que te permite trabajar más en equipo, confiar en los demás y así lograr mejores resultados" (sujeto 11, 20 de julio de 2020).

Storch (2017) afirma que cuando existe evidencia de co-construcción del conocimiento y de apoyo dentro un grupo es cuando el trabajo colaborativo se desarrolla de manera exitosa. Es por ello que la metodología colaborativa puede ser muy útil, siempre y cuando, en la planificación de una actividad en conjunto, se consideren y controlen todos los elementos que se requieren para el logro de las metas; entre ellos, el andamiaje y la interdependencia positiva.

Por otra parte, en relación con los resultados de la escala Likert de apreciación aplicada, se observa que el estudiantado, en su mayoría, destaca de la corrección colaborativa de errores la posibilidad de disipar dudas, de aprender de los compañeros y compañeras, y que, además, puede ser una buena opción para un trabajo o tarea que se realiza comúnmente de manera individual. A su vez, en la revisión de la encuesta, se aprecia que hay un 
punto donde el estudiantado difiere, y es aquel relacionado con la efectividad de la corrección colaborativa contra la corrección del profesorado. En este aspecto, las personas entrevistadas, en su mayoría, no están muy de acuerdo con esta aseveración. Esto demuestra la importancia que sigue teniendo el profesorado en la sala de clases. Lo anterior es compartido por Riddiford (2006), quien declara que la corrección colaborativa de errores no logra reemplazar completamente la corrección del profesorado y que, idealmente, este tipo de tareas necesita corrección por parte de este. Además, es importante señalar que no siempre el profesorado promueve el trabajo colaborativo en la corrección de errores, dado que esto sigue siendo considerado como una responsabilidad del personal docente.

\section{CONCLUSIONES}

Los resultados y su posterior discusión permiten concluir que, en general, la actitud del estudiantado hacia la revisión colaborativa de errores resulta ser positiva. El grupo menciona varias ventajas, entre las que destacan: aprender de las otras personas, que es una de las características que más valoran, dado que permite el intercambio de conocimiento, ideas y opiniones que enriquecen el aprendizaje de cada integrante de un grupo; disipar dudas, donde el estudiantado pueda usarse entre sí como fuente de conocimiento; discutir sobre un tema, que, según el estudiantado, permite una mayor libertad que cuando se está frente al profesorado; optimizar los tiempos de trabajo, dado que se asignan responsabilidades y se dividen tareas; finalmente, promover la autonomía entre estudiantes, puesto que adquieren un rol más activo como parte de la construcción de su propio aprendizaje. Por otra parte, se puede concluir que el estudiantado distingue algunas desventajas de la revisión colaborativa de errores que están relacionadas con el nivel de competencia en inglés del alumnado y de la confianza que exista dentro del grupo. Por lo tanto, estos son aspectos que deben ser considerados por el profesorado al llevar a cabo una tarea colaborativa.

Finalmente, es importante señalar que la metodología colaborativa requiere de una planificación adecuada para que pueda llevarse a cabo de manera satisfactoria. Para este fin es necesario, por una parte, la aplicación adecuada de las técnicas y estrategias que esta metodología ofrece (Barkley et al., 2014), y, por otra, el control y monitoreo constante de la tarea por parte del personal docente, que cumple un rol de facilitador durante la ejecución de la tarea.

\section{ReFERENCIAS}

Assbeihat, J. M. (2016). The impact of collaboration among members on team's performance. Management and Administrative Sciences Review, 5(5), 248-259. https://n9.cl/6e2hkv

Balcarcel, G. M. (2006). La corrección del error en clases de inglés como lengua extranjera. Letras, 48(73), 359-363. https://n9.cl/m2y0j

Baker, M. J. (2015). Collaboration in collaborative learning. Interaction studies, 16, 451-473. https://www.researchg ate.net/publication/288886296_Collaboration_in_collaborative_learning

Barkley, E. F., Cross, K. P. y Major, C. H. (2014). Collaborative learning techniques: A handbook for college faculty. John Wiley y Sons.

Blanco, A. I. (2002). El error en el proceso de aprendizaje. Cuadernos Cervantes de la lengua española, 8(38), 12-22. h ttp://www.cuadernoscervantes.com/art_38_error.html

Bruner, J.S. (1980). The Social Context of Language Acquisition: Witkin Memorial Lecture. Educational Testing Services.

Bruner, J.S. (1983). Child's Talk: Learning to Use Language. Norton.

Delmastro, A. L. (2008). El andamiaje docente en el desarrollo de la lectura y la escritura en lengua extranjera. Paradigma, 29(1), 197-230. 
Ellis, R. (2009). Task-Based Language Teaching Sorting out the Misunderstandings. International Journal of Applied Linguistics, 19(3), 221-246.

Fernández, A. M. y Blum, A. (2013). Collaborative writing in pairs and small groups: Learners' attitudes and perceptions. System, 41(2), 365-378. https://doi.org/10.1016/j.system.2013.02.002

Ferreiro, E. M. (2004). Alfabetización. Teoría y práctica. (6ta ed.). Siglo XXI Editores.

Ferris, D. R. (2013). What L2 writing means to me: Texts, writers, contexts. Journal of Second Language Writing, 22(4), 428-429. https://doi.org/10.1016/j.jslw.2013.08.002

Hernández, R., Fernández, C. B. y Baptista, L. P. (2014). Metodología de la investigación (5ta ed.). McGraw-Hill Interamericana.

Hyland, K. (2000). Disciplinary Discourses. Social Interaction in Academic Writing. Longman.

Li, M. y Storch, N. (2017). Second language writing in the age of CMC: Affordances, multimodality, and collaboration. Journal of Second Language Writing, 36, 1-5. https://n9.cl/5a4l2

Li, M. y Zhu, W. (2017). Good or bad collaborative wiki writing: Exploring links between group interactions and writing products. Journal of Second Language Writing, 35, 38-53. https://n9.cl/wwlqr

Miao, Y., Badger, R. y Zhen, Y. (2016). A comparative study of peer and teacher feedback in Chinese EFL writing class. Journal of Second Language Writing, 15, 179-200. https://doi.org/10.1016/j.jslw.2006.09.004

Ministerio de Educación (Mineduc) (2014). Estándares orientados para carreras de pedagogía en inglés. https://biblio tecadigital.mineduc.cl/bitstream/handle/20.500.12365/2237/mono-596.pdf?sequence=1\&isAllowed=y

Nassaji, H. (2017). Diversity of research methods and strategies in language teaching research. Language Teaching Research 21(2), 140-163. https://journals.sagepub.com/doi/pdf/10.1177/1362168817693696

Ocando, Y. y Urdaneta, M. (2015). El papel de la interferencia en los errores de los aprendices de una segunda lengua. Redhecs, 20, 128-144. https://dialnet.unirioja.es/servlet/articulo?codigo $=6844564$

Pardo, C. y Carrillo, A. C. (2016). L2 Collaborative E-writing. Social and Behavioral Sciences, 22(8), 601-607. https: //www.researchgate.net/publication/305889133_L2_Collaborative_E-writing

Riddiford, N. (2006). Collaborative error correction: How does it help? Prospect, 21(3), 26-37. http://www.ameprc. mq.edu.au/_data/assets/pdf_file/0005/229883/21_3_2_Riddiford.pdf

Storch, N. (2017). Implementing and assessing collaborative writing activities in EAP classes. En J., Bitchener, N Storch y R. Wette, (Eds.), Teaching writingfor academicpurposes to multilingualstudents: Instructional approaches (pp. 130-144). Routledge.

Swain, M. (2000). The output hypothesis and beyond: Mediating acquisition through collaborative dialogue. Sociocultural theory and second language learning, 1, 97-114. https://www.researchgate.net/publication/28410 6490_The_output_hypothesis_and_beyond_Mediating_acquisition_through_collaborative_dialogue

Vygotsky, L. S. (1978). Mind in society: The development of higher psychological processes. Harvard University Press.

Zhang, M. (2018). Collaborative writing in the EFL classroom: The effects of L1 and L2 use. System, 76, 1-12. https://www.researchgate.net/publication/326744634_Collaborative_writing_in_the_EFL_classro om_The_effects_of_L1_and_L2_use

\section{Notas}

1 Este artículo es resultado del proyecto de investigación titulado: Fondecyt 11150273: Uso de estrategias de feedback correctivo escrito focalizado en un entorno virtual colaborativo wiki: Impacto y plan de acción.

2 This paper is the result of the research Project entitled: Fondecyt 11150273: Uso de estrategias de feedback correctivo escrito focalizado en un entorno virtual colaborativo wiki: Impacto y plan de acción.

\section{INFORMACIÓN ADICIONAL}


Mabel Ortiz Navarrete, et al. Actitud del estudiantado universitario hacia la revisión colaborativ...

Cómo citar: Ortiz-Navarrete, M. y Salazar-Betancur, L. (2022). Actitud del estudiantado universitario hacia la revisión colaborativa de errores. Revista Educación, 46(1). http://doi.org/10.15517/revedu.v46i1.45197 